\title{
Relativistic Electron-Cyclotron Waves in a Hot Plasma Channel with a Parabolic Density Profile
}

\author{
E. HEIDARI ${ }^{a * *}$ AND M. AslaninEJAD ${ }^{b}$ \\ ${ }^{a}$ Department of Sciences, Bushehr Branch, Islamic Azad University, Bushehr, Iran \\ ${ }^{b}$ Plasma Physics Research Centre, Science and Research Branch, Islamic Azad University, Tehran, Iran \\ The propagation of relativistic electron-cyclotron waves in a hot plasma channel with a parabolic density profile \\ is discussed. Analytical calculations are performed to find the governing equation for the evolution of the pulse \\ spot size including the effects of relativistic self-focusing, ponderomotive self-channeling and preformed channel \\ focusing. Also, by analyzing the equation of the pulse spot size, the existence of some solitary wave solutions is \\ predicted numerically.
}

DOI: 10.12693/APhysPolA.123.285

PACS: $52.35 . \mathrm{Hr}$

\section{Introduction}

The theoretical and experimental study of nonlinear interactions of intense laser pulses with plasma is an active topic of research. It has potential applications, such as particle and photon acceleration $[1,2]$ and fast ignition in inertial confinement fusion [3]. Relativistic solitons are electromagnetic structures, self-trapped by locally modified plasma refractive index, due to relativistic electron mass increase, and the electron density redistribution by the ponderomotive force of an intense laser pulse. These solitons are generated behind the front of the laser pulse. They are made of spatially localized low-frequency nonlinear electromagnetic (EM) field with a close to zero group velocity. A fairly large part of the laser pulse energy can be transformed to solitons. Recently, the research on solitons has received much attention because they are of fundamental importance in nonlinear sciences [4] and are considered to be essential components of turbulence in plasma [5]. The mechanism of the electromagnetic soliton formation and its structure were analytically investigated and observed by particle simulation in the interaction of intense laser radiation with under and over-dense plasmas [6].

It is well known that a laser pulse in vacuum will diffract over a distance of a Rayleigh length. In other words, the characteristic distance for propagation of a directed radiation beam in vacuum is the Rayleigh range, $Z_{\mathrm{R}}$. In a medium the intensity-dependent, nonlinear, dielectric properties modify this characteristic length $[7,8]$. This can extend the propagation distance compared to that in free space. In a uniform plasma, a laser pulse can guide itself by the effect of relativistic self-focusing and ponderomotive self channeling. However, if the laser power is smaller than the critical power $P_{\mathrm{c}}=17\left(\omega_{0} / \omega_{\mathrm{p}}\right)^{2} \mathrm{GW}$ where $\omega_{0}$ and $\omega_{\mathrm{p}}$ are the laser and plasma frequencies, respectively [9-11], the diffrac-

*corresponding author; e-mail: ehphys75@iaubushehr.ac.ir tion would dominate over the forgoing effects and the laser pulse will diffract again. On the other hand, a preformed plasma channel can prevent diffraction and allow the propagation of the laser pulse through many Rayleigh lengths without disruptions [12-15]. Plasma channels are required to guide the laser light. It has been shown that the beam spot size performs periodic oscillations along the propagation distance in the presence of preformed channel and has equilibrium solution (i.e., constant spot size) when the laser power is equal to the matched powers evaluated with different nonlinear effects [16-18].

In this paper, we investigate on large amplitude relativistic electron-cyclotron waves produced by an intense laser pulse propagating in a hot plasma channel with a parabolic density profile. In Sect. 2, considering the appropriate assumptions, we present the differential equation describing the evolution of the spot size. In Sect. 3, by analyzing the governing equation, we discuss and predict the existence of electromagnetic solitary waves. Results of the numerical solutions are also presented in Sect. 3. Finally in Sect. 4, we summarize the findings of the paper.

\section{Evolution equations}

We assume a circularly polarized laser pulse propagating along the $z$-direction in a hot plasma channel which has a parabolic density profile. We can write the wave equation as [9]:

$$
\begin{aligned}
& \left(\nabla^{2}-\frac{1}{c^{2}} \frac{\partial^{2}}{\partial t^{2}}\right) \boldsymbol{a} \\
& \quad=k_{\mathrm{p}}^{2}\left(1+\frac{r^{2}}{r_{c h}^{2}}-\frac{|\boldsymbol{a}|^{2}}{2}+\nabla_{\perp}^{2} \frac{|\boldsymbol{a}|^{2}}{2}\right) \boldsymbol{a},
\end{aligned}
$$

where $k_{\mathrm{p}}=\omega_{\mathrm{p}} / c$ is the plasma wave number. The second, third and fourth terms on the right hand side of Eq. (1) are due to preformed channeling focus, self-focusing and ponderomotive self-channeling, respectively. Now, assuming that Eq. (1) has a solution with the Gaussian transverse profile [19-22]: 


$$
a(r, t)=a_{r}(z) \mathrm{e}^{-r^{2} / r_{s}^{2}(z)} \mathrm{e}^{\left|b(z) r^{2}+\phi(z)\right|}
$$

and employing the variational method $[9,19]$, we obtain

$$
\begin{aligned}
& \frac{\partial}{\partial z}\left(a_{r} r_{s}\right)=0, \\
& k_{0} \frac{\partial r_{s}}{\partial z}=2 b r_{s}, \\
& k_{0} \frac{\partial b}{\partial z}=\frac{2}{r_{s}^{4}}-2 b^{2}-\frac{k_{\mathrm{p}}^{2}}{2 r_{c h}^{2}}-\frac{a_{r}^{2}}{r_{s}^{4}}-\frac{k_{\mathrm{p}}^{2} a_{r}^{2}}{8 r_{s}^{2}}, \\
& k_{0} \frac{\partial \phi}{\partial z}=-\frac{2}{r_{s}^{2}}+\frac{a_{r}^{2}}{r_{s}^{2}}+\frac{3 k_{\mathrm{p}}^{2} a_{r}^{2}}{16} .
\end{aligned}
$$

From Eqs. (3)-(6) we can obtain the propagation characteristics of the laser pulse in the plasma channel. Combining Eqs. (4) and (6), the normalized equation describing the evolution of the spot size is given as

$$
\frac{\partial^{2} r_{s}}{\partial z^{2}}=\frac{1-p}{r_{s}^{3}}-N_{\mathrm{c}} r_{s}-\frac{a_{0}^{2}}{2 r_{s}^{5}}
$$

Now, for the sake of simplicity, a collimated incident laser pulse is considered. With the initial condition $r_{s}=1$ at $z=0$, integrating Eq. (7) once gives

$$
\frac{1}{2}\left(\frac{\partial r_{s}}{\partial z}\right)^{2}+V\left(r_{s}\right)=0,
$$

where

$$
\begin{aligned}
& V\left(r_{s}\right)=\frac{1}{2} \gamma(1-p) \Lambda\left[r_{s}(\beta-v)\right]^{-2}(1-\beta v) \\
& +\frac{1}{2} \gamma^{2} v^{2} N_{c} r_{s}^{2} \Lambda^{3}[(\beta-v)]^{-2}-\frac{1}{8} \Lambda^{2} a_{0}^{2} r_{s}^{-4}-V_{0}
\end{aligned}
$$

in which

$$
V_{0}=\frac{1}{2} \gamma(1-p) \Lambda \frac{1-\beta v}{(\beta-v)^{2}}+\frac{1}{2} \frac{\gamma^{2} v^{2} N_{\mathrm{c}} \Lambda^{3}}{(\beta-v)^{2}}-\frac{\Lambda^{2} a_{0}^{2}}{8}
$$

and

$$
\Lambda=\frac{e^{2}}{m_{\mathrm{e}}^{2} c^{2}\left(\omega-\omega_{c e}\right)}, \quad \omega_{c e}=\frac{e B_{0}}{m_{\mathrm{e}}} p=k_{\mathrm{p}}^{2} a_{0}^{2} r_{0}^{2} / 16
$$

is the normalized laser power by the critical power $p_{\mathrm{c}}=$ $16 \pi$ for self-focusing in plasma and $N_{\mathrm{c}}=k_{\mathrm{p}}^{2} r_{0}^{4} / 4 r_{c h}^{2}$ is a parameter relating to the effect of preformed channel focusing. Also, $\beta=\frac{u}{c}$ and $v=\frac{V}{c}$ in which $u$ and $V$ are fluid and solitary wave velocities, respectively.

\section{Existence of solitary waves}

Now we turn our attention to investigate the existence of solitary wave solutions of Eq. (8). We follow the same technique used in [22]. The roots of $V\left(r_{s}\right)=0$ can be easily found using the solutions of a cubic equation. The roots, in terms of the coefficient can be expressed as

$$
\begin{aligned}
& r_{s 1}=1, \\
& r_{s 2}=\left[\left(N_{\mathrm{p}}+\sqrt{N_{\mathrm{p}}^{2}-16 N_{\mathrm{c}} a_{0}^{2}}\right) / 8 N_{\mathrm{c}}\right]^{1 / 2}, \\
& r_{s 3}=\left[\left(N_{\mathrm{p}}-\sqrt{N_{\mathrm{p}}^{2}-16 N_{\mathrm{c}} a_{0}^{2}}\right) / 8 N_{\mathrm{c}}\right]^{1 / 2},
\end{aligned}
$$

where

$$
N_{\mathrm{p}}=4(1-p) \Lambda-a_{0}^{2} .
$$

Real and positive roots are acceptable. Different cases can be considered as:

(a) if $p>1-\Lambda^{2} a_{0}^{2} \sqrt{N_{\mathrm{c}}}-\Lambda a_{0}^{2} / 4$, the equation has one real (i.e. $r_{s 1}=1$ ) and two non real complex conjugate roots;

(b) if $p<1-\Lambda^{2}\left(N_{\mathrm{c}}-a_{0}^{2} / 2\right), V\left(r_{s}\right)=0$ has three distinct real roots $r_{s 3}<r_{s 1}=1<r_{s 2}$;

(c) if $1-\Lambda^{2}\left(N_{\mathrm{c}}-a_{0}^{2} / 2\right)<p<1-\Lambda^{2} a_{0}^{2} \sqrt{N_{\mathrm{c}}}-\Lambda a_{0}^{2} / 4$, three cases can be considered:

(c1) if $N_{\mathrm{c}}=N_{\mathrm{c}}^{*}$, where the critical channel parameter $N_{\mathrm{c}}^{*}=\gamma_{0}^{2} a_{0}^{2} / 4, V\left(r_{s}\right)=0$ has triple root, i.e., $r_{s 1}=r_{s 2}=$ $r_{s 3}=1$

(c2) if $N_{\mathrm{c}}>N_{\mathrm{c}}^{*}$, three types can be discussed as follows:

(c2.1) if $p=1-\Lambda^{2} a_{0}^{2} \sqrt{N_{\mathrm{c}}}-\Lambda a_{0}^{2} / 4, V\left(r_{s}\right)=0$ has three real roots: $r_{s 1}=1$ and twofold root $r_{s 2}=r_{s 3}=$ $\sqrt{a_{0} / 2 \sqrt{N_{\mathrm{c}}}}<1$;

$(\mathrm{c} 2.2)$ if $1-\Lambda_{0}^{2}\left(N_{\mathrm{c}}-a_{0}^{2} / 2\right)<p<1-\Lambda^{2} a_{0}^{2} \sqrt{N_{\mathrm{c}}}-\Lambda a_{0}^{2} / 4$, $V\left(r_{s}\right)=0$ has three unequal real roots: $r_{s 3}<r_{s 2}<$ $r_{s 1}=1$;

(c2.3) if $p=1-\gamma_{0}^{2}\left(N_{\mathrm{c}}-a_{0}^{2} / 2\right)$, then $V\left(r_{s}\right)=0$ has three real roots: twofold root $r_{s 1}=r_{s 2}=1$ and $r_{s 3}<1$;

(c3) if $N_{\mathrm{c}}<N_{\mathrm{c}}^{*}$, the following results are given:

(c.3.1) if $p=1-\Lambda^{2} a_{0}^{2} \sqrt{N_{\mathrm{c}}}-\Lambda a_{0}^{2} / 4, V\left(r_{s}\right)=0$ has three real roots: $r_{s 1}=1$ and

$$
r_{s 2}=r_{s 3}=\sqrt{a_{0} / 2 \sqrt{N_{\mathrm{c}}}}>1
$$

(c3.2) if $1-\Lambda_{0}^{2}\left(N_{\mathrm{c}}-a_{0}^{2} / 2\right)<p<1-\Lambda^{2} a_{0}^{2} \sqrt{N_{\mathrm{c}}}-\Lambda a_{0}^{2} / 4$, $V\left(r_{s}\right)=0$ has three unequal real roots: $r_{s 1}=1<r_{s 3}<$ $r_{s 2}$;

(c3.3) if $p=1-\gamma_{0}^{2}\left(N_{\mathrm{c}}-a_{0}^{2} / 2\right), V\left(r_{s}\right)=0$ has three real roots: twofold root $r_{s 1}=r_{s 3}=1$ and $r_{s 2}>1$.

Figures 1-9 show the variations of the potential $V$ for various values of $p$ and $N_{\mathrm{c}}$ corresponding to the cases (a)-(c3.3) respectively. In all cases, fix parameters, $v=$ $0.3, \beta=0.6(\gamma=1.25)$ and $a_{0}=0.3\left(N_{\mathrm{c}}=0.04\right)$ are considered.

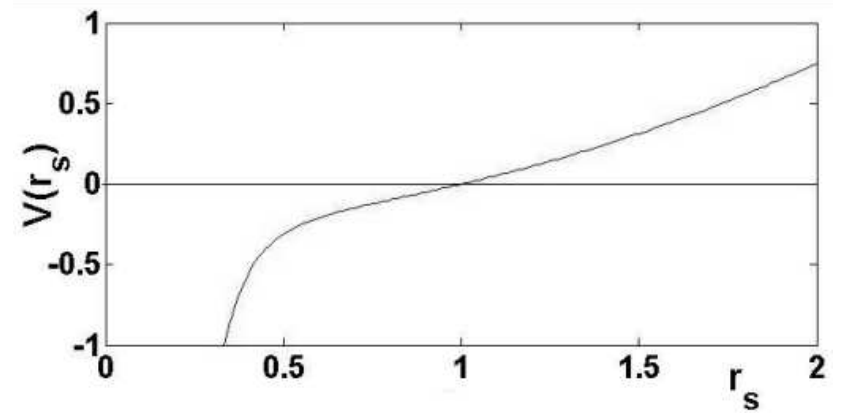

Fig. 1. Potential $V\left(r_{s}\right)$ as a function of spot size $r_{s}$ for $N_{\mathrm{c}}=0.5, p=0.97 . r_{s}=1$ is an unstable position and the particle will move to the position $r_{s} \rightarrow 0$.

As is clear from Figs. 1, 3, 7, 8, $r_{s}=1$ is an unstable position and the particle will move to the position $r_{s} \rightarrow 0$ for the certain parameters introduced in these cases. In fact, these cases correspond to the catastrophic focusing. In Fig. 6, the position $r_{s}=1$ is stable and the particle will be at rest in this case. In fact, this case could be 


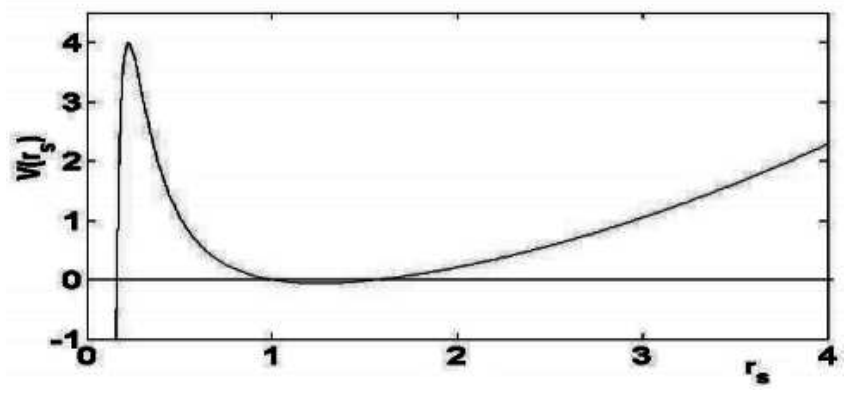

Fig. 2. Potential $V\left(r_{s}\right)$ as a function of spot size $r_{s}$ for $N_{\mathrm{c}}=0.5, p=0.2$. Particle will move periodically between $r_{s 1}$ and $r_{s 2}$.

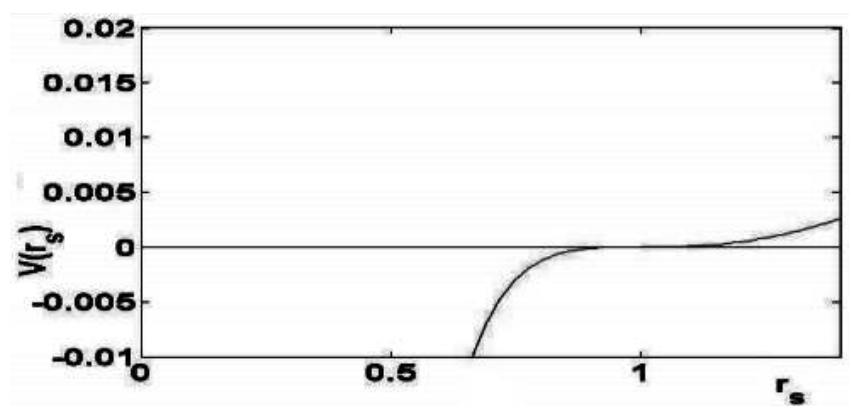

Fig. 3. Potential $V\left(r_{s}\right)$ as a function of spot size $r_{s}$ for $N_{\mathrm{c}}=0.04, p=1.04 . r_{s}=1$ is an unstable position and the particle will move to the position $r_{s} \rightarrow 0$.

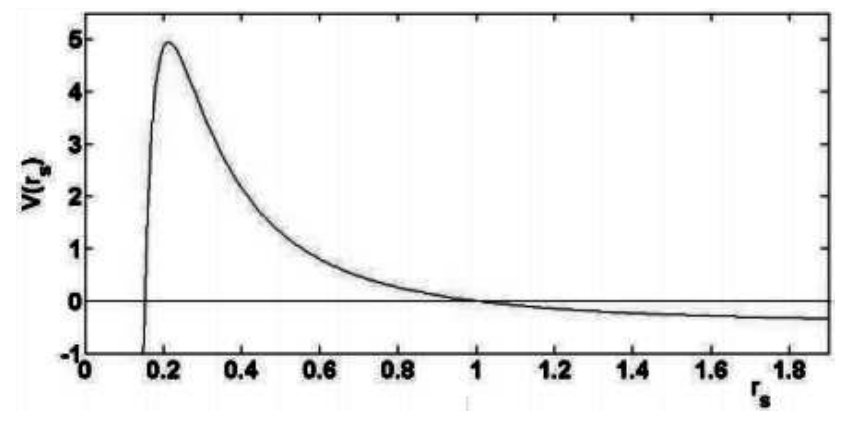

Fig. 4. Potential $V\left(r_{s}\right)$ as a function of spot size $r_{s}$ for $N_{\mathrm{c}}=0.5, p=0.796$. Particle is in critical state.

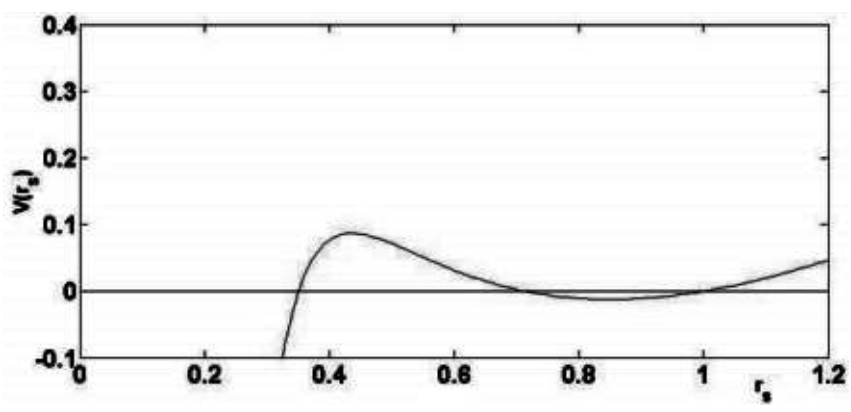

Fig. 5. Potential $V\left(r_{s}\right)$ as a function of spot size $r_{s}$ for $N_{\mathrm{c}}=0.5, p=0.65$. Particle will move periodically between $r_{s 1}$ and $r_{s 2}$.

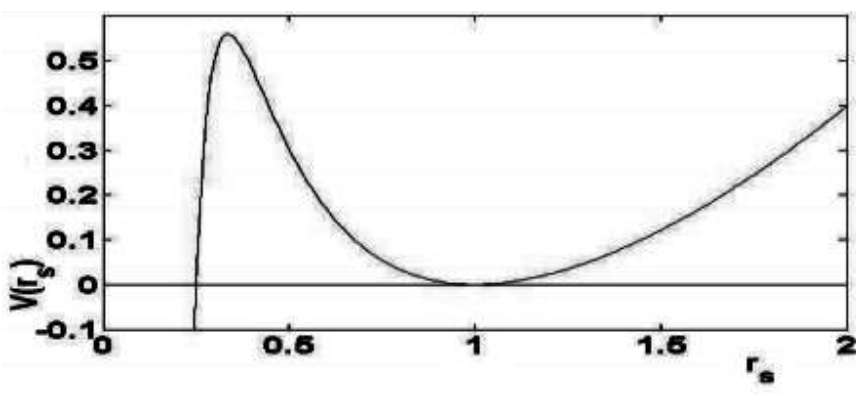

Fig. 6. Potential $V\left(r_{s}\right)$ as a function of spot size $r_{s}$ for $N_{\mathrm{c}}=0.5, p=0.34375$. Position $r_{s}=1$ is stable and the particle will be at rest.

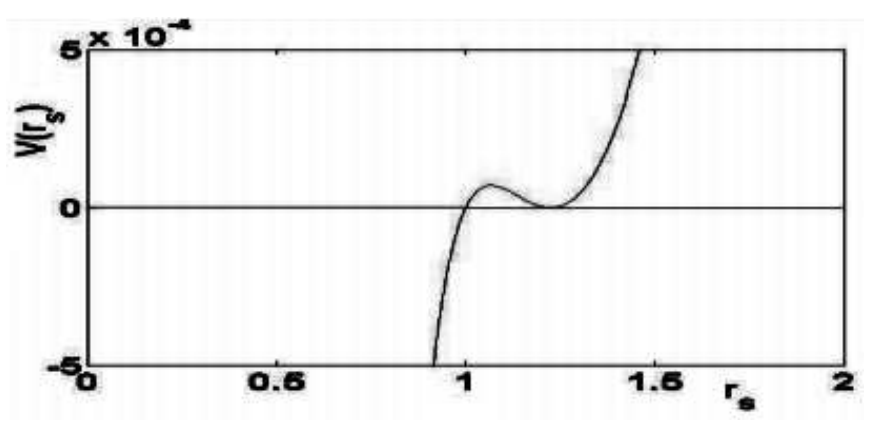

Fig. 7. Potential $V\left(r_{s}\right)$ as a function of spot size $r_{s}$ for $N_{\mathrm{c}}=0.02, p=0.9092 . r_{s}=1$ is an unstable position and the particle will move to the position $r_{s} \rightarrow 0$.

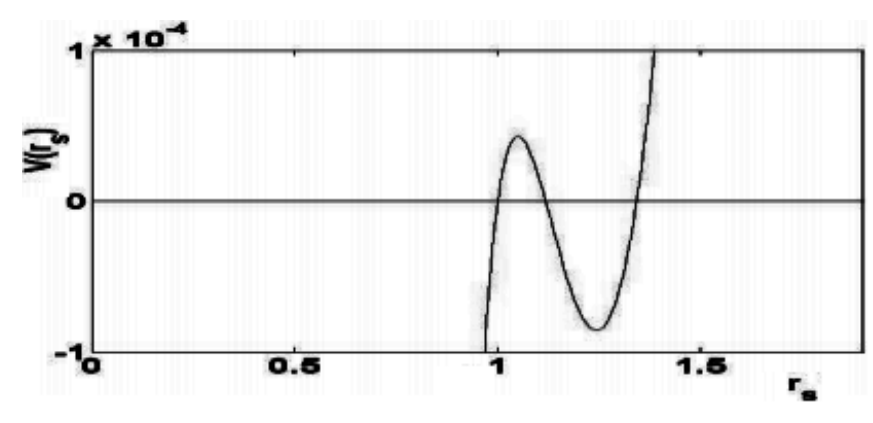

Fig. 8. Potential $V\left(r_{s}\right)$ as a function of spot size $r_{s}$ for $N_{\mathrm{c}}=0.02, p=0.998 . r_{s}=1$ is an unstable position and the particle will move to the position $r_{s} \rightarrow 0$.

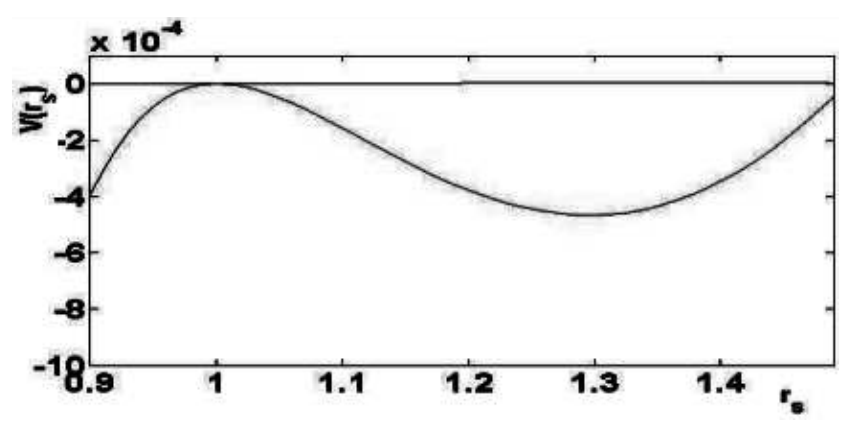

Fig. 9. Potential $V\left(r_{s}\right)$ as a function of spot size $r_{s}$ for $N_{\mathrm{c}}=0.02, p=1.09375$. Particle is in critical state. 
related to a constant spot size. In Figs. 2 and 5, particle will move periodically between $r_{s 1}$ and $r_{s 2}$ which shows the characteristic feature of periodic solutions. Finally, Figs. 4 and 9 indicated that the particle is in critical state.

\section{Summary}

Using the appropriate equations, we presented the governing equation of the laser spot size. By analyzing the differential equation, we discussed and obtained the solutions of the potential function. Also, we presented the potential evolutions with the spot size.

\section{Acknowledgments}

This research paper has been financially supported by the office of vice chancellor for research of Islamic Azad University, Bushehr Branch.

\section{References}

[1] P.K. Kaw, J.M. Dawson, Phys. Fluids 13, 472 (1970).

[2] T. Tajima, J.M. Dawson, Phys. Rep. 122, 173 (1985)

[3] H. Abbasi, M.R. Rouhani, D.D. Teskhakaya, Phys. Scr. 56, 619 (1997)

[4] A.V. Gurevich, J. Exp. Theor. Phys. 53, 953 (1967).

[5] N.N. Rao, p.K. Shukla, M.Y. Yu, Phys. Fluids 27, 2664 (1984)

[6] L.M. Kerashvili, N.L. Tsintsadze, Fiz. Plaszmy 9, 570 (1983).
[7] W.G. Wagner, H.A. Haus, J.H. Marburger, Phys. Rev. 175, 256 (1968)

[8] E.L. Dawes, J.H. Marburger, Phys. Rev. 179, 862 (1969).

[9] S. Zhang, B.S. Xie, X.R. Hong, H.C. Wu, X.Y. Zhao, Phys. Plasmas 18, 033104 (2011)

[10] C.E. Max, J. Arons, A.B. Langdon, Phys. Rev. Lett. 33, 209 (1974)

[11] P. Sprangle, E. Esarey, A. Ting, G. Joyce, Appl. Phys. Lett. 53, 2146 (1988)

[12] E. Esarey, p. Sprangle, J. Krall, A. Ting, IEEE Trans. Plasma Sci. 24, 252 (1996)

[13] B. Hafizi, A. Ting, p. Sprangle, R.F. Hubbard, Phys. Rev. E 62, 4120 (2000)

[14] B. Hafizi, A. Ting, R.F. Hubbard, p. Sprangle, J.R. Peano, Phys. Plasmas 10, 1483 (2003)

[15] B.J. Duda, W.B. Mori, Phys. Rev. E 61, 1925 (2000)

[16] E. Esarey, p. Sprangle, J. Krall, A. Ting, IEEE Trans. Plasma Sci. 24, 252 (1996)

[17] H. Tang, H. Guo, Phys. Lett. A 321, 111 (2004)

[18] M. Liu, H. Guo, D. Deng, Y. Zhang, X. Chen, Acta Phys. Sin. 5, 1419 (2004) (in Chinese).

[19] E. Heidari, M. Aslaninejad, H. Eshraghi, Plasma Phys. Control. Fusion 52, 075010 (2010).

[20] P.K. Shukla, B. Eliasson, Phys. Rev. Lett. 94, 065002 (2005)

[21] B. Eliasson, p.K. Shukla, JETP Lett. 83, 447 (2006).

[22] B. Eliasson, p.K. Shukla, Phys. Lett A 354, 453 (2006). 